\title{
Effect of temperature on longitudinal optical phonon-assisted exciton luminescence in heteroepitaxial GaN layer
}

\author{
S. J. Xua) \\ Department of Physics, The University of Hong Kong, Pokfulam Road, Hong Kong, China \\ W. Liu and M. F. Li \\ Department of Electrical Engineering, National University of Singapore, 10 Kent Ridge Crescent, \\ Singapore 119260, Singapore
}

(Received 10 January 2000; accepted for publication 22 September 2000)

\begin{abstract}
First-order and second-order longitudinal optical (LO) phonon-assisted luminescence lines of neutral donor-bound excitons and free excitons in heteroepitaxial GaN film have been investigated in temperature range from 3.5 to $50 \mathrm{~K}$. The energy spacing between the exciton resonant lines and their corresponding LO phonon replicas is found to be strong temperature dependent. Permogorov's theory on LO phonon-assisted luminescence of free excitons could be employed to explain the main experiments. It is also found that the interaction strength of the free exciton with LO phonon is stronger than that of the bound exciton with LO phonon. (C) 2000 American Institute of Physics. [S0003-6951(00)04047-X]
\end{abstract}

The shallow neutral donor-bound exciton emission (usually noted as $D^{0}, X$, or $I_{2}$ ) often dominates in lowtemperature photoluminescence spectrum of heteroepitaxial GaN films. Currently, the highest quality heteroepitaxial GaN films grown by metalorganic vapor phase epitaxy (MOVPE) have $D_{0}, X$ linewidths between 2 and $3 \mathrm{meV}$ at low temperature. ${ }^{1}$ In the heteroepitaxial thick GaN films grown by hydride vapor phase epitaxy (HVPE) the neutral donor-bound exciton linewidths are as narrow as about one unit electron volt. As a result, a rich variety of fine structure is resolved, for example, two or more neutral donor-bound exciton transitions have been identified. ${ }^{1,2}$ On the other hand, as a polar semiconductor, exciton-longitudinal optical (LO) phonon coupling in $\mathrm{GaN}$ is strong enough to permit that the phonon-assisted exciton luminescence lines can be observed..$^{3-6}$ In the literature ${ }^{3-5}$ the LO phonon-assisted freeexciton luminescence in the epitaxial $\mathrm{GaN}$ films has been investigated. Reynolds et al. ${ }^{6}$ observed the phonon sidebands of the donor-bound exciton transition in the relatively thick GaN epilayers grown by HVPE. In this letter, we present the first-order and second-order LO phonon-assisted luminescence lines of the neutral donor-bound excitons and the free excitons in the heteroepitaxial GaN film at different temperatures from 3.5 to $50 \mathrm{~K}$. It is found that the energy separations between the phonon sidebands and their principle lines exhibit unusual temperature dependence. Moreover, the twophonon energy spacing shows a significantly smaller decrease with temperature from 3.5 to $50 \mathrm{~K}$ than the onephonon energy spacing.

The sample used in the experiment was grown on $c$ plane of sapphire by MOVPE. A $2.88 \mu \mathrm{m} \mathrm{GaN} \mathrm{layer} \mathrm{was}$ grown followed a $40 \mathrm{~nm}$ GaN nucleation layer. The sample is nominally undoped. In the photoluminescence (PL) measurements, the sample was mounted on the cold finger of a Janis closed cycle cryostat with varying temperature from

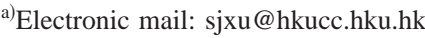

3.5 to $300 \mathrm{~K}$, and excited by the $325 \mathrm{~nm}$ line of a Kimmon $\mathrm{He}-\mathrm{Cd}$ continuous wave laser with output power of $40 \mathrm{~mW}$. The emission signal was dispersed by a SPEX 750M monochromator and detected with a Hamamatsu R928 photomultiplier. The lock-in amplifier technology was not used because of strong enough emission signal.

The exciton PL spectra of the sample at different temperatures are shown in Fig. 1. Clearly, the neutral donorbound exciton $\left(D^{0}, X\right)$ line dominates in the examined lowtemperature range. Its linewidth is $2.4 \mathrm{meV}$ under low power excitation, indicating high quality of the sample. In strainrelaxed GaN samples (homoepitaxial films, ${ }^{7}$ or thick heteroepitaxial films $)^{2,8}$ the $D^{0}, X$ peak occurs at $3.471 \mathrm{eV}$ at low temperature. In our sample, the $D^{0}, X$ peak is at $3.490 \mathrm{eV}$ at $4 \mathrm{~K}$, indicating a large compressive, biaxial stress, which we estimate to be $1.2 \mathrm{GPa}$. The free exciton $A$ transition (FXA) is also well resolved. Besides the $D^{0}, X$ peak and FXA transition, one pair of weaker peaks with energy $\sim 20 \mathrm{meV}$ lower

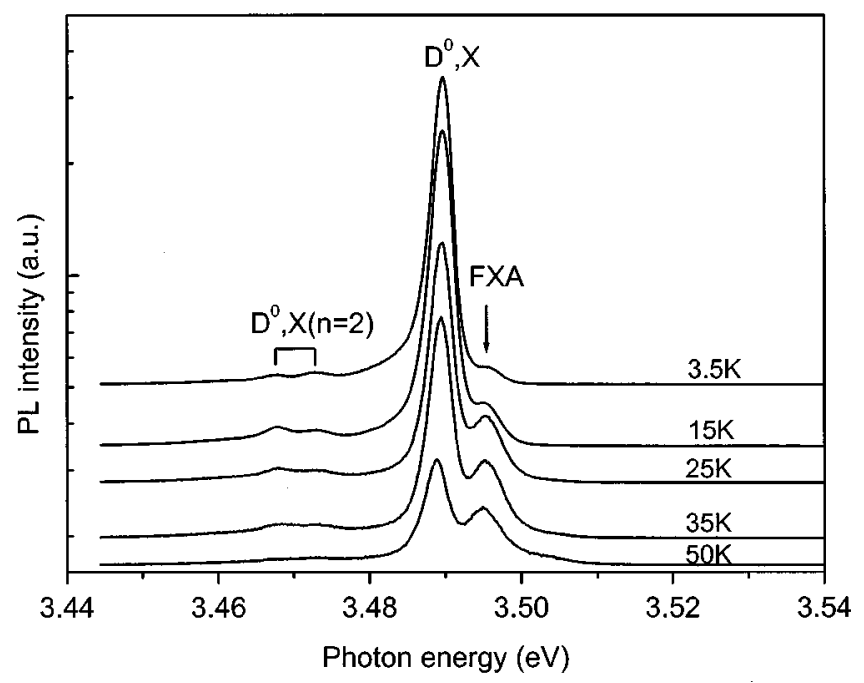

FIG. 1. The exciton resonant luminescence spectra of the GaN epilayer at different temperatures. 


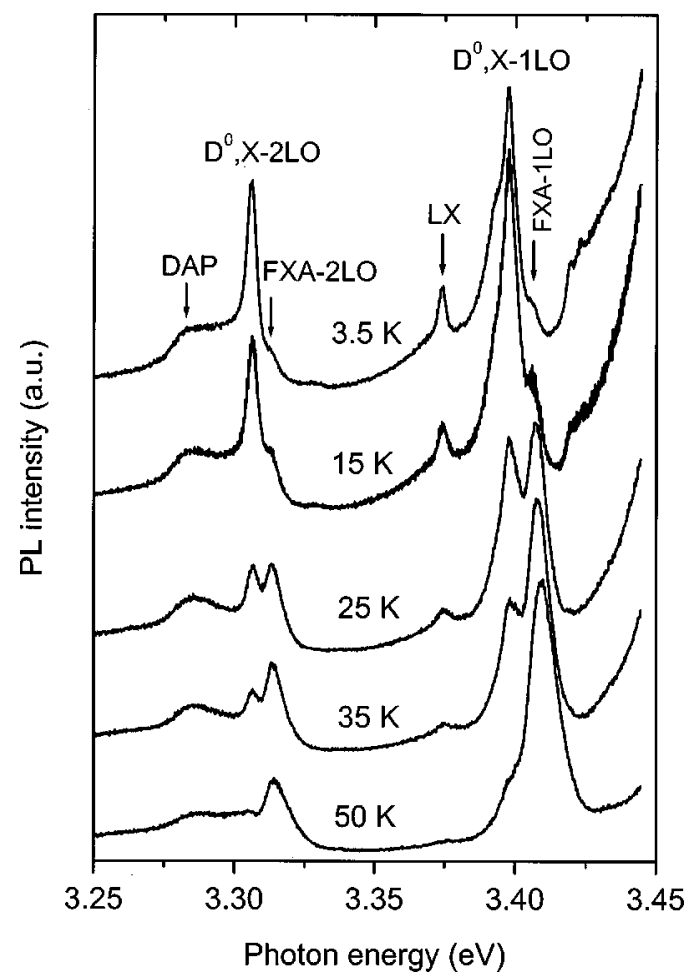

FIG. 2. The LO phonon-assisted luminescence spectra measured at different temperatures.

than that of the $D^{0}, X$ line are observed. They have been recently identified to be the excited state transitions [denoted by $\left.D^{0}, X(n=2)\right]$ of the $D^{0}, X$ using magnetospectroscopy technique by Skromme et al. ${ }^{2,9,10}$ Actually, existence of the large number of excited states is an important feature of the shallow donor-bound excitons in direct gap III-V and II-VI compound semiconductors. ${ }^{11}$

As temperature increases, the relative intensity of the FXA line with respect to the $D^{0}, X$ line rapidly increases. This is obviously due to thermal dissociation of the bound excitons into the free excitons. ${ }^{3}$ However, $D^{0}, X$ transition still keeps its dominant position in the interested temperature range. The relative intensity of the phonon sidebands of the FXA with respect to the phonon sidebands of the $D^{0}, X$ increases with temperature more significantly than that of the FXA with respect to the $D^{0}, X$, as shown in Fig. 2. At about $25 \mathrm{~K}$, the phonon sidebands of the FXA already become dominant. When the temperature is further increased to $50 \mathrm{~K}$, the phonon sidebands of the $D^{0}, X$ almost disappears. These facts imply that the coupling strength of bound exciton with LO phonon in much weaker than that of free exciton with LO phonon. Relatively broad peak denoted by DAP is the distinct donor-acceptor pair recombination. ${ }^{12}$ Another excitonic transition (LX) may be from the radiative recombination of the excitons localized at extended defects in the GaN. ${ }^{1,13,14}$

It is known that the LO phonon characteristics energy can be determined from the energy spacing between the exciton resonant lines and their LO phonon sidebands. ${ }^{6}$ It comes as a surprise that the energy spacing exhibits an unusual temperature dependence, as shown in Fig. 3. The characteristic energy of $A_{1}-\mathrm{LO}$ phonon of $\mathrm{GaN}$ has been determined to be $\sim 92 \mathrm{meV}$ with low-temperature PL technique. ${ }^{3-6}$ Thus, the dot lines at $92 \mathrm{meV}$ are used as ref-

Downloaded 03 Nov 2006 to 147.8.21.97. Redistribution subject
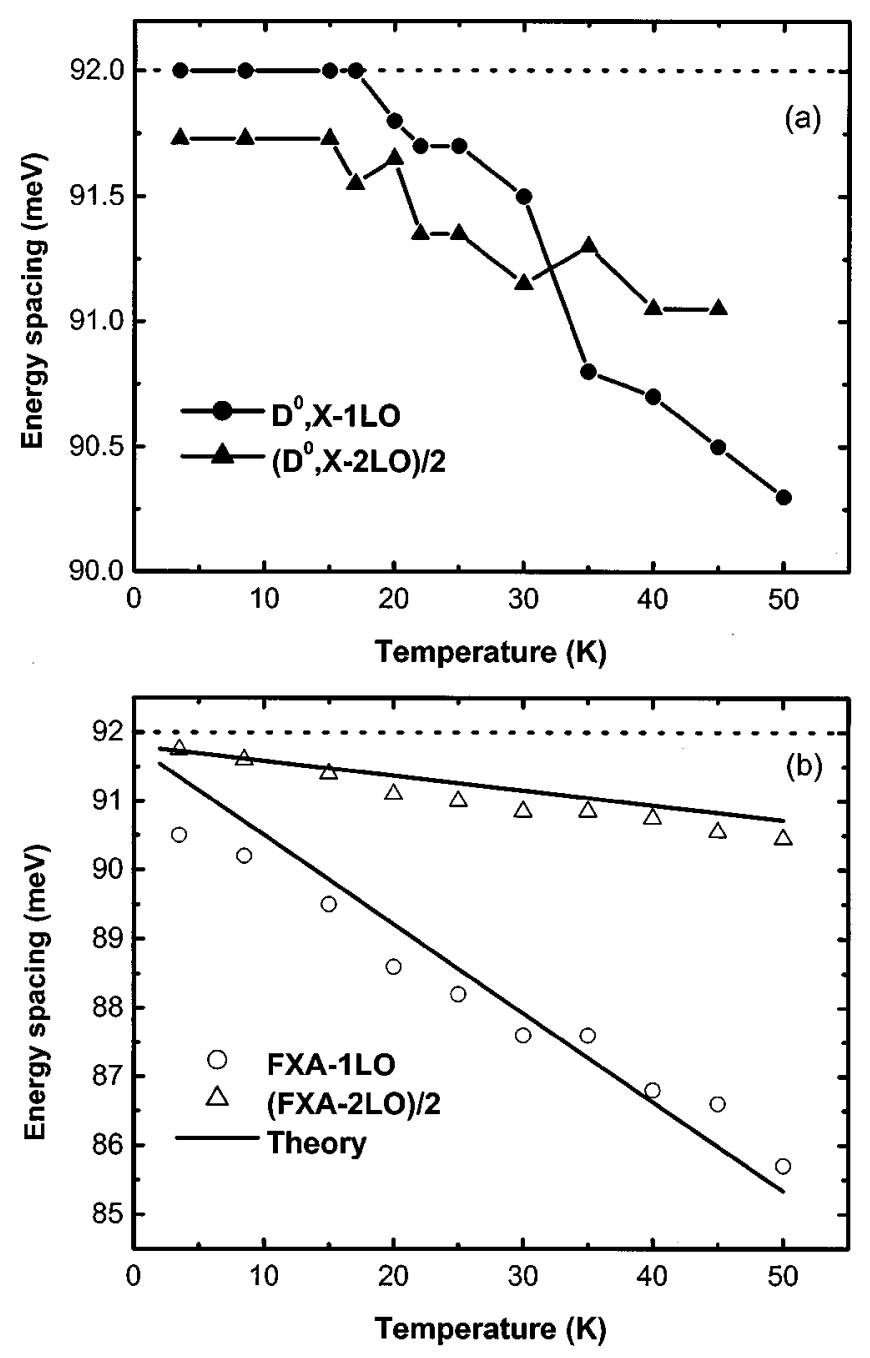

FIG. 3. The $1 \mathrm{LO}$ phonon energy spacing and 2 LO phonon energy spacing versus temperature, (a) determined from donor-bound exciton transitions; (b) determined from free exciton transitions. The solid lines in (a) are guide to eye while the solid lines are the theoretical results in (b). The dot lines at $92 \mathrm{meV}$ in (a) and (b) are plotted as reference lines.

erence lines in Fig. 3. Four physical quantities, denoted by $D^{0}, X-1 \mathrm{LO}, \quad\left(D^{0}, X-2 \mathrm{LO}\right) / 2, \quad$ FXA-1LO, and $(\mathrm{FXA}-2 \mathrm{LO}) / 2$, respectively, were plotted as a function of temperature in Fig. 3. Both the $D^{0}, X-1 \mathrm{LO}$ and the FXA$1 \mathrm{LO}$ exhibit a surprising decrease with temperature. Compared with the $D^{0}, X-1 \mathrm{LO}$ and the FXA-1LO, however, the $\left(D^{0}, X-2 \mathrm{LO}\right) / 2$ and $(\mathrm{FXA}-2 \mathrm{LO}) / 2$ show much weaker temperature dependence. Generally, for the sample investigated here, there are three possible physical mechanisms that can cause shift of the energy separations with temperature. The first factor is lattice thermal expansion due to vibrational anharmonicity of solid atoms or molecules. It is expected that the phonon energy decreases with increasing temperature. Following Liu et al.'s work ${ }^{15}$ on the temperature dependence of the LO phonons of the GaN films, we calculate the shift amount of the $A_{1}-\mathrm{LO}$ phonon frequency of the GaN film for temperature varying from 4 to $50 \mathrm{~K}$ using the Cui's empirical formula. ${ }^{16}$ The shift is found to be only 0.002 $\mathrm{cm}^{-1}$ when the parameters obtained by Liu et al. ${ }^{15}$ were adopted in calculation. Debernardi's first principles calculations ${ }^{17}$ also show a very small shift of the phonon frequency due to anharmonic effect in the interested temAIP license or copyright, see http://apl.aip.org/apl/copyright.jsp 
perature range. Therefore, the thermal expansion should be ruled out as a main factor causing the observable large temperature dependence of the phonon energy in the GaN film.

Another factor causing a shift of the phonon frequency is pressure or stress. ${ }^{18}$ As mentioned earlier, the residual stress in the GaN film investigated in the present work is estimated to be $1.2 \mathrm{GPa}$. Such a large residual stress is mainly due to the thermal expansion coefficient difference between $\mathrm{GaN}$ epilayer and sapphire substrate. ${ }^{8}$ It should be expected that the change in the thermal stress and thus the change in the optical phonon frequency is negligibly small in the temperature range from 4 to $50 \mathrm{~K}$ although the low-temperature thermal coefficients of $\mathrm{GaN}$ are not available so far. ${ }^{19,20}$

The third factor should be from the exciton-phonon coupling mechanism. According to Permogorov's theory ${ }^{21}$ on optical emission due to free exciton scattering by LO phonons in semiconductors, the spectral shape of the phononassisted luminescence lines can be described by the general formula

$$
J_{N}(\varepsilon)=\varepsilon^{1 / 2} \exp \left(-\varepsilon / k_{B} T\right) W_{N}(\varepsilon),
$$

where $W_{N}(\varepsilon)$ is the probability of the $N$ th order phononassisted annihilation for the exciton with the kinetic energy $\varepsilon$. The spectral lineshape defined by Eq. (1) is a typical asymmetrical lineshape. If $W_{N}$ is a simple power function of the excitonic kinetic energy $W_{N} \propto \varepsilon^{L}$, the halfwidth of Eq. (1) depends linearly on temperature with a slope unambiguously related to the exponent $L$. The more interesting theoretical prediction is that the shift $\Delta$ of the line maximum from its low-energy threshold $(\varepsilon=0)$ also increases linearly with temperature

$$
\Delta=\left(L+\frac{1}{2}\right) k_{B} T .
$$

The shift of the line maximum of phonon-assisted luminescence lines with temperature will doubtless result in reduction of the energy spacing between the zero-phonon line and its phonon replica line. The reduction speed of the energy spacing depends obviously on the exponent $L$. For the case of $1 \mathrm{LO}$ phonon scattering, Premogorov theoretically demonstrated that the probability $W_{1}$ is approximately proportional to the kinetic energy $\varepsilon$. In other words, $L=1$ for the firstorder LO phonon-assisted luminescence. For the secondorder LO phonon-assisted luminescence, it has been found that the annihilation probability is independent of the excitonic kinetic energy, that is, $L=0$. According to earlier analysis, the 1LO energy spacing will linearly decrease with temperature in a slope of $-\frac{3}{2} k_{B}$. The $2 \mathrm{LO}$ energy spacing defined in the present work will also linearly decrease with temperature but in a much smaller slope of $-\frac{1}{4} k_{B}$. The solid lines in Fig. 3(b) are the theoretical results based on the Permogorov's model. In the theoretical calculations, the characteristic energy of $A_{1}-\mathrm{LO}$ phonons at $0 \mathrm{~K}$ was taken as $91.8 \mathrm{meV}$. The agreement between the experiments of free excitons and the theory is very well. However, the temperature of the $D^{0}, X-1 \mathrm{LO}$ is much weaker than that of the FXA-1LO. The change of the $D^{0}, X-1 \mathrm{LO}$ in the examined temperature range is only $1.7 \mathrm{meV} / 92 \mathrm{meV} \approx 1.8 \%$ while the change of the FXA-1LO is $6.3 \mathrm{meV} / 92 \mathrm{meV} \approx 6.8 \%$. This indicates that there is a significant difference in scattering mechanism between free exciton-phonon and bound exciton-phonon. Together with the much stronger temperature dependence of the emission intensity of the 1LO phonon-assisted luminescence line, the much weaker temperature dependence of the $D^{0}, X-1 \mathrm{LO}$ indicates that the coupling strength of the bound excitons with the LO phonons is much smaller than that of the free excitons.

In summary, the LO phonon-assisted luminescence of the shallow donor-bound excitons and free excitons in the heteroepitaxial $\mathrm{GaN}$ film has been investigated at different temperatures. Anomalous temperature dependence of the energy separation between the principal exciton resonant lines and their responding phonon replicas was found. Moreover, $1 \mathrm{LO}$ phonon spacing exhibits much stronger temperature dependence than $2 \mathrm{LO}$ phonon spacing does. It was found that the Permogorov's theory could be employed to interpret our main experiments.

This project is financially supported by the program of light-emitting materials at HKU.

${ }^{1}$ B. K. Meyer, A. Hoffmann, and P. Thurian, in Group III Nitride Semiconductor Compounds: Physics and Applications, edited by B. Gil (Oxford, UK, 1998), Chap. 7, p. 243.

${ }^{2}$ B. J. Skromme, J. Jayapalan, R. P. Vaudo, and V. M. Phanse, Appl. Phys. Lett. 74, 2358 (1999).

${ }^{3}$ D. Kovalev, B. Averboukh, D. Volm, B. K. Meyer, H. Amano, and I. Akasaki, Phys. Rev. B 54, 2518 (1996).

${ }^{4}$ I. A. Buyanova, J. P. Bergman, B. Monemar, H. Amano, and I. Akasaki, Mater. Sci. Eng., B 50, 130 (1997).

${ }^{5}$ W. Liu, M. F. Li, S. J. Xu, K. Uchida, and K. Matsumoto, Semicond. Sci. Technol. 13, 769 (1998).

${ }^{6}$ D. C. Reynolds, D. C. Look, B. Jogai, and R. J. Molnar, Solid State Commun. 108, 49 (1998).

${ }^{7}$ K. Kornitzer, T. Ebner, K. Thonke, R. Sauer, V. Kirchner, V. Schwegler, M. Kamp, M. Leszcynski, I. Grzegory, and S. Porowski, Phys. Rev. B 60, 1471 (1999).

${ }^{8}$ D. Volm, K. Oettinger, T. Streibl, D. Kovalev, M. Ben-Chorin, J. Diener, B. K. Meyer, J. Majewski, L. Eckey, A. Hoffman, H. Amano, I. Akasaki, K. Hiramatsu, and T. Detchprohm, Phys. Rev. B 53, 16543 (1996).

${ }^{9}$ B. Gil, O. Briot, and R.-L. Aulombard, Phys. Rev. B 52, R17028 (1995).

${ }^{10}$ B. J. Skromme, H. Zhao, B. Goldenberg, H. S. Kong, M. T. Leonard, G. E. Bulman, C. R. Abernathy, and S. J. Pearton, in III-V Nitrides, edited by F. A. Ponce, T. D. Moustakas, I. Akasaki, and B. A. Monemar (Materials Research Society, Pittsburgh, 1997), Vol. 449, p. 173.

${ }^{11}$ P. J. Dean and D. C. Herbert, in Excitons, edited by K. Cho (Springer, Berlin, 1979), p. 55.

${ }^{12}$ M. Leroux, N. Grandjean, B. Beaumont, G. Nataf, F. Semond, J. Massies, and P. Gibart, J. Appl. Phys. 86, 3721 (1999).

${ }^{13}$ C. Wetzel, S. Fischer, J. Krüger, E. E. Haller, R. J. Molnar, T. D. Moustakas, E. N. Mokhov, and P. G. Baranov, Appl. Phys. Lett. 68, 2556 (1996).

${ }^{14}$ L. Eckey, J.-Ch. Holst, P. Maxim, R. Heitz, A. Hoffmann, I. Broser, B. K. Meyer, C. Wetzel, E. N. Mokhov, and P. G. Baranov, Appl. Phys. Lett. 68, 415 (1996).

${ }^{15}$ M. S. Liu, L. A. Bursill, S. Prawer, K. W. Nugent, Y. Z. Tong, and G. Y. Zhang, Appl. Phys. Lett. 74, 3125 (1999).

${ }^{16}$ J. B. Cui, K. Amtmann, J. Ristein, and L. Ley, J. Appl. Phys. 83, 7929 (1998).

${ }^{17}$ A. Debernardi, Solid State Commun. 113, 1 (2000).

${ }^{18}$ B. A. Weinstein and R. Zallen, in Light Scattering in Solid IV, edited by M. Cardona and G. Güntherodt (Springer, Berlin, 1984), Chap. 8, p. 463.

${ }^{19}$ Instrinsic Properties of Group IV Elements and II-V, II-VI, and I-VII Compounds, Landolt-Börnstein Vol. 22, Pt. a, edited by O. Madelung, M. Schulz, and H. Weiss (Springer, Berlin, 1982), p. 181.

${ }^{20}$ G. K. White, in Thermal Expansion of Solids, edited by C. Y. Cho (ASM International, USA, 1998), Chap. 11, p. 272.

${ }^{21}$ S. Permogorov, in Excitons, edited by E. I. Rashba and M. D. Sturge (North-Holland, Amsterdam, 1982), Chap. 5. 